\title{
UTILIZAÇÃO DO PROCESSO DE ENFERMAGEM E AS DIFICULDADES ENCONTRADAS POR ENFERMEIROS
}

\author{
Vanessa Soares da Silva1ㄹ, Euclides Sales Barbosa Filho1', Samia Mara Barros de Queiroz², \\ Rita Neuma Dantas Cavalcante de Abreu ${ }^{3}$
}

\begin{abstract}
RESUMO: Objetivou-se averiguar a importância atribuída à utilização do processo de enfermagem por enfermeiros e identificar as principais dificuldades encontradas. Estudo qualitativo, descritivo, realizado em $2011 \mathrm{em}$ um hospital de Fortaleza-Ceará, com 9 enfermeiros, e cuja coleta dos dados foi realizada por meio de entrevista não estruturada. A partir da análise dos resultados originaram-se três categorias: Importância da utilização do Processo de Enfermagem durante a assistência aos clientes; Utilização das fases do processo durante a assistência de enfermagem e Dificuldades encontradas pelos enfermeiros na utilização do Processo de Enfermagem. Conclui-se que, apesar dos enfermeiros considerarem a sistematização da assistência um instrumento para sua qualificação, esses referem dissociação de suas etapas, déficit de conhecimento e de quantitativo de profissionais como dificuldades a sua aplicabilidade.
\end{abstract}

DESCRITORES: Enfermagem; Processo de enfermagem; Neurologia; Sistematização da assistência de enfermagem.

\section{USE OF THE NURSING PROCESS AND DIFFICULTIES FOUND BY NURSES}

ABSTRACT: This study aimed to check the importance attributed to the use of the nursing process by nurses and to identify the main difficulties found. The study was qualitative and descriptive, and was undertaken in 2011 in a hospital in FortalezaCeará, with 9 nurses, and data collection was carried out through non-structured interviews. Based on analysis of the results, three categories were produced: Importance of the use of the Nursing Process during assistance to clients; Use of the stages of the process during nursing care and Difficulties found by the nurses in using the Nursing Process. It is concluded that in spite of the nurses' considering the systematization of care an instrument for their training, they mention dissociation of its stages, inadequate knowledge, and inadequate staff numbers as difficulties in applying it.

DESCRIPTORS: Nursing; Nursing process; Neurology; Systematization of nursing care.

\section{UTILIZACIÓN DEL PROCESO DE ENFERMERÍA Y LAS DIFICULTADES ENCONTRADAS POR ENFERMEROS}

RESUMEN: La finalidad del estudio fue averiguar la importancia atribuida a la utilización del proceso de enfermería por enfermeros e identificar las principales dificultades. Estudio cualitativo, descriptivo, realizado en 2011 en un hospital de Fortaleza, Ceará, con nueve enfermeros. Los datos fueron obtenidos por medio de entrevista no estructurada. Con base en el análisis de los resultados, surgieron tres categorías: Importancia de la utilización del Proceso de enfermería durante la asistencia a los clientes; Utilización de las fases del proceso durante la asistencia de enfermería y Dificultades encontradas por los enfermeros en la utilización del Proceso de enfermería. Se concluye que, a pesar de que los enfermeros consideraron la sistematización de la asistencia un instrumento para su cualificación, eses relatan disociación de sus etapas, déficit de conocimiento y de cuantitativo de profesionales como dificultades a su aplicabilidad.

DESCRIPTORES: Enfermería; Proceso de enfermería; Neurología; Sistematización de la asistencia de enfermería.

\footnotetext{
${ }^{1}$ Enfermeiro.

${ }^{2}$ Enfermeira do Instituto Dr. José Frota - IJF e do Hospital Nossa Senhora da Conceição. Especialista em Enfermagem Obstétrica e em Educação em Saúde. Membro do Grupo de Pesquisa Saberes em Práticas de Enfermagem em Emergência - SEMPRE. Membro do Grupo de Pesquisa Cuidado Clínico do Idoso e as Práticas Educativas - GRUPESS da Universidade Estadual do Ceará - UECE.

${ }^{3}$ Enfermeira do Instituto Dr. José Frota IJF. Mestre em Cuidados Clínicos. Doutoranda em Biotecnologia pela UECE. Professora do Curso de Graduação em Enfermagem da Universidade de Fortaleza - UNIFOR. Membro do SEMPRE - UNIFOR.
} 


\section{INTRODUÇÃO}

As doenças neurológicas podem ser estáveis ou progressivas, caracterizar-se por períodos assintomáticos e também por flutuações nos sintomas. A história de saúde inclui, portanto, detalhes a respeito do início, natureza, gravidade, localização, duração e frequência dos sinais e sintomas; queixas associadas; fatores precipitantes, agravantes e de alívio; evolução, remissão e exacerbação; e a presença ou ausência de sintomas semelhantes em membros da família ${ }^{(1)}$.

Os enfermeiros precisam ser hábeis na avaliação do sistema neurológico. Esta exige um conhecimento de anatomia e da fisiologia do sistema nervoso e uma compreensão da gama de testes e procedimentos usados no cuidado aos clientes com alterações neste sistema $^{(1)}$. A utilização de documentação precisa de diagnósticos de enfermagem é fundamental para enfermeiros na prática hospitalar diária, objetivando planejar corretamente, implementar, bem como avaliar os cuidados de enfermagem para os indivíduos ${ }^{(2)}$.

A Enfermagem vem aprimorando seus conhecimentos e propondo novas alternativas de assistência, desenvolvendo metodologia própria de trabalho, fundamentada no método científico, isto é, fundamentada no processo de enfermagem $(\mathrm{PE})^{(2)}$. Ressalta-se a utilização do PE durante a assistência aos clientes com doenças neurológicas orienta o trabalho do enfermeiro para coletar dados, identificar as necessidades de cuidados, propor intervenções e avaliar os resultados dos cuidados que realiza. Assim, a produção do conhecimento científico sobre a temática reveste-se de fundamental importância para avaliação da assistência de enfermagem ${ }^{(3)}$.

Existem várias etapas envolvidas no processo de identificação do problema/necessidade. O PE prevê que a assistência seja pautada na avaliação do paciente, que fornece os dados para que os diagnósticos sejam identificados, os quais direcionam a definição de metas a serem alcançadas. Juntos, diagnósticos e metas, são as bases para selecionar as intervenções mais apropriadas à situação específica do paciente. Realizadas as intervenções, o alcance das metas deve ser avaliado e dessa avaliação retorna-se às fases precedentes, caso as metas não tenham sido alcançadas, ou novos diagnósticos tenham sido identificados ${ }^{(4)}$. Portanto, 0 PE se operacionaliza em etapas: coleta de dados de enfermagem ou histórico de enfermagem; diagnóstico de enfermagem; planejamento de enfermagem; implementação e avaliação de enfermagem.
A coleta de dados de enfermagem consiste no levantamento de informações referentes ao estado de saúde do cliente, da família e da comunidade, com propósito de identificar as necessidades, os problemas, as preocupações e as reações humanas desse cliente. $\mathrm{O}$ exame físico é um procedimento a ser realizado no dia-a-dia das atividades do enfermeiro, como forma de proporcionar informações sobre as capacidades funcionais do paciente, e utilizado na elaboração dos diagnósticos de enfermagem, na determinação das intervenções a serem realizadas, bem como na avaliação da efetividade dos cuidados prestados, permitindo, dessa forma, a individualização da assistência ${ }^{(5)}$.

O diagnóstico de enfermagem é caracterizado como um julgamento clínico sobre as respostas do indivíduo, da família ou da comunidade aos problemas reais ou de risco para a saúde/processos vitais; se constitui como a base para a seleção das prescrições que propiciarão o alcance dos resultados pelos quais a enfermeira é responsável ${ }^{(6)}$. Após a análise do diagnóstico, examinando as necessidades afetadas e o grau de dependência do cliente, planeja-se os cuidados a serem prestados. A quarta fase, implementação, norteará a equipe de enfermagem na realização dos cuidados e a avaliação dos mesmos fornecendo os dados necessários para a quinta fase. A avaliação de enfermagem se baseia na avaliação diária das adaptações do paciente diante dos cuidados prestados. Através da avaliação de enfermagem, é possível avaliar se os cuidados realizados pela equipe de enfermagem estão oferecendo benefícios ao paciente, oportunizando a real independência da assistência de enfermagem ${ }^{(7)}$.

O PE deve ser estabelecido na prática em todas as instituições de saúde, em hospitais, assim como na saúde comunitária como um todo. A sistematização de cuidados enfermagem é obrigatória e imprescindível nas instituições, através da resolução 358/2009 do COFEN, artigo primeiro, que dispões que o $\mathrm{PE}$ deve ser realizado, de modo deliberado e sistemático, em todos os ambientes em que ocorre o cuidado profissional de enfermagem ${ }^{(8)}$.

Este estudo faz parte de um projeto maior sobre a Sistematização da Assistência de Enfermagem (SAE) aos portadores de distúrbios neurológicos. Porém, para que a SAE seja implantada no hospital do estudo é necessário investigar a importância atribuída pelos enfermeiros ao processo. Assim, têm-se como objetivos: averiguar a importância atribuída na utilização do processo de enfermagem pelos enfermeiros e identificar as principais dificuldades encontradas por estes profissionais ao utilizar o processo no cuidado aos clientes. 


\section{MÉTODO}

Estudo de natureza qualitativa, do tipo descritivo, realizado em um hospital de Fortaleza-Ceará. $\mathrm{O}$ atendimento prestado por este serviço tem caráter ambulatorial, cirúrgico, emergencial e eletivo. Este funciona diuturnamente, nos fins de semana e feriados com plantonistas no local de atendimento, preferentemente, à população através do Sistema Único de Saúde (SUS), muito embora atenda também à Rede Suplementar de Saúde e particulares. Neste hospital há duas unidades destinadas aos pacientes portadores de distúrbios neurológicos diversos.

A população do estudo foi composta por 15 enfermeiros de ambos os sexos que cuidam de pessoas com distúrbios neurológicos, funcionários do hospital em estudo. Foram excluídos os enfermeiros que, tendo concordado em participar, não devolveram o instrumento de coleta de dados, finalizando 9 participantes.

A coleta dos dados foi realizada por intermédio de entrevista com perguntas abertas. $\mathrm{O}$ instrumento foi elaborado constando de questões relacionadas à sistematização da assistência de enfermagem: Você conhece as fases do processo de enfermagem? Quais? Você considera importante a utilização do processo de enfermagem durante a assistência aos portadores de distúrbios neurológicos? Por quê? Durante a assistência de Enfermagem você aplica alguma fase do Processo de Enfermagem? Você tem alguma dificuldade em utilizar o processo de enfermagem?

A coleta de dados iniciou-se em agosto de 2011 e encerrou-se em outubro de 2011, a análise foi baseada no método de análise de conteúdo ${ }^{(9)}$, configurando-se um conjunto de técnicas de análise das comunicações que utiliza procedimentos sistemáticos e objetivos de descrição do conteúdo das mensagens, composto das seguintes etapas: Constituição do corpus; Composição das unidades de análise; Codificação e recortes; Categorização; Descrição das categorias.

A discussão dos resultados foi realizada por meio da utilização de literatura pertinente no que concerne ao PE. Utilizou-se as letras do alfabeto (de A a I) para designar os enfermeiros visando garantir o anonimato. $\mathrm{O}$ projeto foi encaminhado ao Comitê de Ética em Pesquisa da Universidade de Fortaleza tendo sido aprovado sob o número 005/11. Os princípios éticos foram seguidos em todas as fases do estudo, em consonância com o que preconiza a Resolução 196/96 que dispõe sobre a ética de pesquisa envolvendo seres humanos ${ }^{(10)}$. Uma cópia do projeto foi encaminhada ao Diretor e à Coordenadora de Enfermagem do Hospital, tendo sido autorizado.

\section{RESULTADOS}

Os 9 participantes da pesquisa encontravam-se na faixa etária entre 24 e 41 anos, com predomínio da idade entre 31 e 41 anos. Em relação ao tempo de formação profissional, quatro participantes tinham de três meses a um ano de formação e cinco tinham entre dois e onze anos; e sete eram especialistas.

Concluídas as etapas da análise de conteúdo, as falas deram origem a três categorias, a seguir apresentadas.

\section{Importância da utilização do Processo de Enferma- gem durante a assistência aos clientes}

Quatro enfermeiros afirmaram que a assistência às necessidades do cliente e a elaboração do plano de cuidados individualizado são de bastante relevância durante o atendimento ao paciente, como recorte das falas a seguir:

Tem fundamental importância [o Processo de Enfermagem] porque a partir do processo é que prepara o esquema do plano de cuidados. Para que haja a continuidade e a avaliação do enfermeiro relacionado ao cuidado. (Enf. D)

[...] avaliar, organizar e sistematizar a assistência de enfermagem ao cliente. Podemos de forma contínua realizar os cuidados adequados para cada necessidade do cliente. (Enf. B)

Todas são essenciais para elaboração de um plano de cuidado individualizado dentro da necessidade de cada ser. (Enf. A)

O mesmo se torna importante no que se diz respeito ao norteamento da assistência às necessidades do cliente. Os mesmos precisam de uma assistência individualizada e centrada na sua patologia. Iremos ter um cuidado mais direcionado a cada paciente, suprimindo suas necessidades. SAE, cuidado individualizado $e$ humanizado. (Enf. H)

O cuidar holístico foi citado por um enfermeiro:

Todas são essenciais para elaboração de um plano de cuidado [...]. Porque dessa forma o cuidar será mais holístico, diante do distúrbio especifico. (Enf. A)

Os enfermeiros citaram as fases do PE e a importância de cada uma (Quadro 1). 
Quadro 1 - Fases do processo de enfermagem citadas pelos enfermeiros. Fortaleza, 2011

\begin{tabular}{|c|c|}
\hline FASES & RELATOS DOS ENFERMEIROS \\
\hline \multirow{5}{*}{ COLETA DE DADOS DE ENFERMAGEM } & $\begin{array}{l}\text { Identificar informações, famílias, riscos, queixa principal. } \\
\text { Exame físico. (Enf. C) }\end{array}$ \\
\hline & $\begin{array}{l}\text { É um roteiro onde vamos levantar dados sobre o paciente, } \\
\text { exame físico é importante nele, achamos dados perceptiveis. } \\
\text { (Enf. E) }\end{array}$ \\
\hline & $\begin{array}{l}\text { A importância do histórico de enfermagem é um momento de } \\
\text { interação entre profissionais e cliente. (Enf. F) }\end{array}$ \\
\hline & $\begin{array}{l}\text { Torna possivel a identificação dos problemas do paciente, } \\
\text { exame fisico. (Enf. G) }\end{array}$ \\
\hline & Terideiadosurgimento dapatologiaeidentificaros problemas. (Enf.I) \\
\hline \multirow{2}{*}{ DIAGNÓSTICO DE ENFERMAGEM } & $\begin{array}{l}\text { Baseado na NANDA, nele devemos ter analisado os dados colhi- } \\
\text { dos no histórico e exame físico que nos indicará os problemas } \\
\text { de Enfermagem. (Enf. E) }\end{array}$ \\
\hline & $\begin{array}{l}\text { Onde faremos um julgamento clínico sobre as respostas do } \\
\text { indivíduo, prevenção, promoção, recuperação e manutenção } \\
\text { da saúde. (Enf. G) }\end{array}$ \\
\hline PLANEJAMENTO DE ENFERMAGEM & $\begin{array}{l}\text { A importância da prescrição é porque prescreve de acordo com } \\
\text { o diagnóstico de enfermagem. Eu acho que traça um programa } \\
\text { de ações objetivas a partir o diagnóstico de enfermagem. (Enf. F) }\end{array}$ \\
\hline IMPLEMENTAÇÃO & Inclui a execução do cuidado planejado. (Enf. C) \\
\hline \multirow{5}{*}{ AVALIAÇÃO DE ENFERMAGEM } & $\begin{array}{l}\text { Análise dos cuidados prestados e processo da doença, ou seja, me- } \\
\text { lhora do paciente. Parâmetro da melhora dos agravos diariamente. } \\
\text { (Enf. C) }\end{array}$ \\
\hline & $\begin{array}{l}\text { É um documento importante, avalia as condições gerais do paciente, } \\
\text { necessário para SAE. Nele deve conter todas as anotações ocorridas } \\
\text { no dia, tornando indispensável na aplicação do processo de Enfer- } \\
\text { magem. (Enf. E) }\end{array}$ \\
\hline & $\begin{array}{l}\text { É importante porque você avalia o paciente como um todo [seu es- } \\
\text { tado geral]. Sim, porque é um conjunto onde você avalia, identifica } \\
\text { necessidades do paciente. (Enf. F) }\end{array}$ \\
\hline & $\begin{array}{l}\text { É um relatório diário para mudanças sucessivas. A utilização do } \\
\text { processo de Enfermagem deve ser mantida a todos os pacientes. } \\
\text { (Enf. G) }\end{array}$ \\
\hline & $\begin{array}{l}\text { Visualizar comoo paciente está. Prognóstico: qual caminho opaciente } \\
\text { irá, sim, pois podemos realizar o plano de cuidados que irá melhorar } \\
\text { as condições clínicas do nosso cliente. (Enf. I) }\end{array}$ \\
\hline
\end{tabular}

Utilização das fases do processo durante a assistência de enfermagem

No que se refere à utilização das fases do PE durante a assistência ao cliente, observou-se que todos os enfermeiros citaram que, em algum momento de suas atividades, usaram de forma parcial ou em sua totalidade, conforme apresentado no quadro 2.
Quadro 2 - Etapas do Processo de Enfermagem utilizadas pelos enfermeiros. Fortaleza, 2011

\begin{tabular}{|l|c|}
\hline Etapas do PE & N \\
\hline Coleta de dados de Enfermagem & 7 \\
\hline Diagnóstico de Enfermagem & 4 \\
\hline Planejamento de Enfermagem & 7 \\
\hline Implementação & 7 \\
\hline Avaliação de Enfermagem & 8 \\
\hline
\end{tabular}




\section{Dificuldades encontradas pelos enfermeiros na utilização do PE}

$\mathrm{Na}$ terceira categoria pode-se destacar que cinco enfermeiros citaram ter dificuldades em utilizar o PE, como alguns relatos a seguir:

A aceitação perante as instituições privadas, devido ao nivel de enfermeiros ser insuficiente e o próprio acumular várias funções, deixando o processo de enfermagem esquecido. (Enf. B)

Aqui no hospital tenho [dificuldades] pois a SAE ainda não foi mantida como documento indispensável para o enfermeiro. (Enf. E)

Resistência dos profissionais; deficit de conhecimento. (Enf. I)

Quatro dos entrevistados não relataram dificuldades em utilizar o PE em suas atividades.

\section{DISCUSSÃO}

No que se refere à importância da utilização do PE durante a assistência aos clientes, sabe-se que a SAE representa um instrumento de trabalho do enfermeiro com objetivo de identificação das necessidades do paciente, apresentando uma proposta ao seu atendimento e cuidado, direcionando a equipe de enfermagem nas ações a serem realizadas. Trata-se de um processo dinâmico, que requer do profissional base científica, conhecimento, habilidades e atitudes pautadas no compromisso ético, na responsabilidade e no assumir o cuidar do outro ${ }^{(11)}$.

$\mathrm{O}$ PE passou a ser empregado como um instrumento de trabalho do enfermeiro, como método sistematizador da sua assistência, que possibilita ao mesmo aplicar na prática o marco teórico que direciona e que embasa as suas ações. Ao mesmo tempo estes buscavam a sua autonomia profissional e o status da profissão ${ }^{(12)}$. A dinâmica organizada da SAE, por meio da sequência das suas fases que estão interrelacionadas e interdependentes, baseadas no conhecimento científico da enfermeira, identifica as necessidades do indivíduo. Através de uma intervenção, proporciona os resultados que se esperam dela, pois, como intervenção terapêutica, o cuidado é centralizado nas necessidades do paciente que devem ser atendidas. A ação será de cuidado, se a enfermeira identifica uma necessidade e sabe o que fazer para atendê-la ${ }^{(13)}$.
Os enfermeiros deste estudo, muitas vezes, se manifestaram como se as fases do processo fossem independentes. Percebeu-se que eles conheciam bem a primeira etapa do processo, porém, poucos se posicionaram nas fases de planejamento de enfermagem e implementação. O planejamento da assistência de enfermagem é um dos meios que o enfermeiro dispõe para aplicar seus conhecimentos técnico-científicos e humanos na assistência ao paciente e caracterizar sua prática profissional, colaborando na definição de seu papel $^{(14)}$. Durante o plano de cuidados, o enfermeiro elabora as ações de enfermagem visando resolver os problemas encontrados durante a fase de diagnósticos. Os cuidados devem ser reavaliados periodicamente com o objetivo de aprimoramento do planejamento das ações ${ }^{(15)}$.

Na etapa da avaliação de enfermagem, foi evidenciado conhecimento dos enfermeiros, podendo estar relacionado à utilização dessa etapa durante a prática assistencial. No entanto, apesar da separação do PE em fases distintas, sabe-se que grande parte dos enfermeiros não aplica na íntegra o processo e, quando o fazem, utilizam com maior frequência a coleta de dados e avaliação de enfermagem, relatando que não conseguem fazer o registro dessas atividades devido ao fator tempo ${ }^{(16)}$.

Por meio do PE o enfermeiro promove cuidado humanizado, dirigido a resultados e de baixo custo e, ainda, esse é capaz de impulsionar os enfermeiros a continuamente examinarem suas práticas, refletindo sobre formas de fazê-las melhor ${ }^{(17)}$. Dentre as fases do processo descritas pelos enfermeiros que participaram da pesquisa, observou-se que, apesar da maioria utilizar quase todas as etapas do processo, a grande dificuldade é a aplicação da fase do diagnóstico de enfermagem. Corrobora-se com alguns autores quando explicam que a identificação dos diagnósticos de enfermagem de um grupo de clientes possibilita o conhecimento das respostas humanas alteradas, contribuindo para o desenvolvimento de intervenções direcionadas e individualizadas. Deste modo, o conhecimento dos problemas de saúde de um grupo de pacientes com características comuns direciona a assistência de enfermagem individualizada, fornecendo subsídios para a elaboração do plano de cuidados, da implementação de intervenções e qualificação da equipe de enfermagem ${ }^{(2)}$.

Apesar dos profissionais terem afirmado conhecer a SAE, foi possível perceber as dificuldades encontradas na utilização desta, como a carência de pessoal. Para pesquisadores os principais fatores que dificultam a implantação da SAE são: a falta de conhecimento para 
a realização do exame físico, a falta de conhecimento sobre o tema nas instituições de saúde, a falta de registro adequado da assistência de enfermagem, conflito de papéis, dificuldades de aceitação de mudanças, falta de credibilidade nas prescrições de enfermagem, carência de pessoal e falta de estabelecimento de prioridades organizacionais ${ }^{(18)}$.

Autores relatam que os profissionais, principalmente os envolvidos nas funções administrativas e gerenciais dos sistemas de cuidados, percebem crescente descompasso entre o conhecimento que é produzido na academia e a prática. Acreditam que esse elemento podem contribuir para a desvalorização da SAE e dificultar o desenvolvimento de processos mais flexíveis e dialógicos na perspectiva da interdisciplinaridade ${ }^{(19)}$.

\section{CONSIDERAÇÕES FINAIS}

Na instituição hospitalar onde foi realizada a pesquisa verificou-se que os enfermeiros acreditam que a SAE constitui um instrumento capaz de melhorar da assistência, embora em alguns momentos foi percebida a separação de cada fase do processo, como se fossem independentes. As dificuldades citadas pelos enfermeiros podem contribuir diretamente para não aplicabilidade do PE.

Este estudo reitera que embora os enfermeiros atribuam valor ao $\mathrm{PE}$, na prática este instrumento de qualificação da assistência de enfermagem permanece pouco empregado na rotina assistencial.

\section{REFERÊNCIAS}

1. Smeltzer SC, Bare BG. Brunner-Suddarth. Tratado de enfermagem médico-cirúrgica. 11a ed. Rio de Janeiro: Guanabara Koogan; 2009.

2. Paans W, Roos MB, Cees N, Schans PV, Sermeus W. What factors influence the prevalence and accuracy of nursing diagnoses documentation in clinical practice? A systematic literature review. J Clinical Nurs. 2011;1(20):2386-403.

3. Duran ECM, Toledo VP. Análise da produção do conhecimento em processo de enfermagem: estudo exploratório-descritivo. Rev. Gaúcha Enferm. 2011;32(2):234-40.

4. Guedes ES, Turrini RNT, Sousa RMC, Baltar VT, Cruz DALM. Atitudes dos profissionais de enfermagem relacionadas ao processo de enfermagem. Rev Esc Enferm USP. 2012;46 (n.esp):130-7.
5. Ohl RIB, Michel JLM, Lopes RS. Exame físico geral. In: Barros ALBL, organizadora. Anamnese e exame físico: avaliação diagnóstica de enfermagem no adulto. Porto Alegre: Artmed; 2003.

6. Nanda. Diagnósticos de Enfermagem da NANDA: definições e classificação 2012-2014. Porto Alegre: Artmed; 2012.

7. Sales LM, Afonso ESR, Santos TVC. Sistematização da assistência de enfermagem (SAE): uma pesquisa nas bases eletrônicas de dados. EMAS. 2008;3(1):197-207.

8. Conselho Federal de Enfermagem. Resolução n. 358, de 15 de outubro 2009. Dispõe sobre a sistematização da assistência de enfermagem e a implementação do processo de enfermagem em ambientes, públicos ou privados em que ocorre o cuidado profissional de enfermagem e dá outras providências. Rio de Janeiro: COFEN; 2009.

9. Bardin L. Análise de conteúdo. 5a ed. Lisboa: Edições 70; 2009.

10. Ministério da Saúde (BR). Conselho Nacional de Saúde. Resolução n. 196/96 - Normas regulamentadoras de pesquisa envolvendo seres humanos. Brasília (Brasil): Ministério da Saúde; 1996.

11. Menezes SRT, Priel MR, Pereira LL. Autonomia e vulnerabilidade do enfermeiro na prática da sistematização da assistência de enfermagem. Rev Esc Enferm USP. 2011;45(4):953-8.

12. Brandalize DL, Kalinowski CE. Processo de enfermagem: vivência na implantação da fase de diagnóstico. Cogitare enferm. 2005;10(3):53-7.

13. Repeto MA. Avaliação da sistematização da assistência de enfermagem - SAE - em um hospital universitário [tese]. São Paulo (SP): UNIFESP; 2003.

14. Pivotto F, Lunardi Filho WD, Lunardi VL. Prescrição de enfermagem: dos motivos da não realização às possíveis estratégias de implementação. Cogitare enferm. 2004;9(2):32-42.

15. Rodrigues MM, Souza MS, Silva JL. Sistematização da assistência de enfermagem na prevenção da lesão tecidual por pressão. Cogitare enferm. 2008;13(4):566-75.

16. Santos MGPS, Medeiros MMR, Gomes FQC, Enders BC. Percepção de enfermeiros sobre o processo de enfermagem: uma integração de estudos qualitativos. Rev Rene. 2012;13(3):712-23. 
17. Álfaro-Lefevre R. Aplicação do processo de enfermagem: promoção do cuidado colaborativo. 5a ed. Porto Alegre: Artmed; 2005.

18. Remizoski L, Rocha MM, Vall J. Dificuldades na implantação da sistematização da assistência de enfermagem - SAE: uma revisão teórica. Cadernos da Escola de Saúde, Curitiba; 2010.

19. Backes DS, Koerich MS, Nascimento KC, Erdmann AL. Nursing care systematization as a multidimensional and interactive phenomenon. Rev. Latino-Am. Enfermagem. 2008;16(6):979-85. 